A. Pramesh Rao, G. Swarup and Gopal-Krishna, eds.

\title{
Physical Conditions in CSS Radio Sources
}

\author{
S. A. Tyul'bashev and P. A. Tchernikov \\ Pushchino Radio Astronomy Observatory, Astro Space Center of the \\ Lebedev Institute of Physics, Russian Academy of Sciences, Pushchino, \\ Moscow 142292, Russia
}

\begin{abstract}
Interplanetary scintillation observations of 56 compact steepspectrum radio sources have been carried out at $102 \mathrm{MHz}$ on the Large Phased Array. Observations have shown that 42 sources have low frequency cut-offs in their spectra. The physical conditions in the compact components of the quasars and radio galaxies studied are estimated.
\end{abstract}

\section{Introduction}

Compact steep-spectrum (CSS) radio sources is a separate class of sources with steep spectra $\left(\alpha>0.5, S \sim \nu^{-\alpha}\right)$ and angular sizes $<1^{\prime \prime}$. The linear dimensions of these sources turned out to be smaller than those of the host galaxies. Optical identifications indicate that, as a rule, these sources are quasars and radio galaxies. Many CSS sources have been observed with high angular resolution at centimeter and decimeter wavelengths. At the same time, information about the physical conditions in their cores is supplied by the low-frequency cut-offs of their spectra, which most often occur at meter wavelengths. This leads to the necessity of low-frequency observations of CSS sources. The aim of the study presented here is to investigate the physical conditions in the nuclei of the galaxies hosting compact radio sources with steep spectra at high frequencies.

\section{Observations}

For our study, we chose 56 CSS radio sources from Sanghera et al. (1995) which have been well studied at high frequencies including VLBI studies. 28 sources are galaxies, 26 quasars and 2 have no optical identification.

We carried out $102 \mathrm{MHz}$ interplanetary scintillation observations in 19951997 on the Large Phased Array of the Lebedev Institute of Physics, Russia. The effective area of the antenna in the zenith direction is $3 \times 10^{4} \mathrm{~m}^{2}$, the receiver time constant $\tau=0.4 \mathrm{~s}$, and the bandwidth is about $200 \mathrm{kHz}$. The rms confusion due to extended (nonscintillating) sources is $\sim 1 \mathrm{Jy}$. The data reduction method is given in Artyukh \& Tyul'bashev (1996). This method enables us to detect faint scintillating sources, for which the scintillation fluctuation is smaller than the noise fluctuation. Scintillations were observed for all sources. Estimates of the integrated flux densities and of compact (scintillating) component were obtained. The structure of each source from the VLBI-maps published in literature have been thoroughly investigated. Such analysis allows us to reveal one or several 
components dominating at $102 \mathrm{MHz}$, to obtain their correct frequency spectra and to estimate their angular sizes.

\section{Physical Conditions in the Sources Studied}

Our observations have shown that 42 of 56 sources studied have low-frequency cut-offs in their spectra. Assuming that the low-frequency cut-offs in the spectra are the result of synchrotron self-absorption, we have estimated the magnetic field strength, relativistic plasma density and the energies of magnetic field and of relativistic plasma in the compact components of 42 sources from the sample studied using the method published in Artykh (1988). The acccuracy of this approach is one order of magnitude. We emphasize that the method used for estimating these physical parameters does not assume equipartition between the energies of the magnetic field and of the relativistic plasma.

Most part of sources have magnetic field strength in the range $10^{-2} \mathrm{G}$ to $10^{-5} \mathrm{G}$. The energies of magnetic field significantly differ from the energies of relativistic particles. Energy equipartition is observed in 8 of 42 sources. 2 of which are galaxies and 6 are quasars. 11 galaxies and 9 quasars have the energy of magnetic field several orders of magnitude higher than the energy of relativistic particles. 3 galaxies and 11 quasars show opposite situation. Thus, most of the galaxies have dominating energy in magnetic fields, while quasars have different balances between energies, including equipartition. There is a possible correlation between linear sizes and physical parameters of compact components. The relativistic particles density increases on the lower scales, at the same time magnetic field strength increases on the greater scales. But these dependences may be doubtful because of the errors in estimates of physical parameters.

We find no evidence for correlations between magnetic field strength, relativistic particles density and source redshift. Therefore, there seems to be no evidence for evolution of the parameters until $z \sim 2-3$. However, the reason of this result may be due to the fact that the nature of the sources of our sample is significantly different (hot spots, jets, cores, radio lobes, etc.).

It was found that 5 galaxies and 1 quasar have $10^{-1} \mathrm{G}<H<1 \mathrm{G}, 9$ galaxies and 5 quasars have $10^{-3} \mathrm{G}<H<10^{-2} \mathrm{G}, 3$ galaxies and 8 quasars have $10^{-5} \mathrm{G}<H<10^{-4} \mathrm{G}$. For other sources the derived values are not reliable. The mean magnetic field strength in quasars $\left(\sim 10^{-4} \mathrm{G}\right)$ seems to be weaker than mean magnetic field strength of galaxies $\left(\sim 10^{-3} \mathrm{G}\right)$.

\section{References}

Artyukh, V. S. 1988, Works of Lebedev Phys. Inst., 189, 223

Sanghera, H., Saikia, D., Ludke, E., Spencer, R., Foulsham, P., Akujor, C., Tzioumis, A. 1995, AAp, 295, 629

Artyukh, V. S., \& Tyul'bashev, S. A. 1996, AZh, 73, 669 